\title{
PREVALÊNCIA DE KLEBSIELLA SPP. ESBL ISOLADA EM HOSPITAL ESCOLA DO SUL DE MINAS GERAIS
}

\author{
${ }^{1}$ Mauy MANA \\ ${ }^{1}$ Natália BONASSI \\ ${ }^{1}$ Sabrina ROMANELLI \\ ${ }^{2}$ Terezinha Inêz Estivalet SVIDZINSK \\ ${ }^{3}$ Raquel Maria Lima LEMES \\ 1. Acadêmica de Medicina da Faculdade de Medicina de Itajubá - FMIt \\ 3. Professora Doutora Associado do Departamento de Análises Clínicas, Universidade Estadual de Maringá - UEM, \\ 3. Professora Doutora Adjunto II do Departamento de Análises Clínicas e Toxicológicas da FCF da UNIFAL-MG, \\ raquel.lemes@unifal-mg.edu.br \\ *AUTOR PARA CORRESPONDENCIA: Dra. Raquel Maria Lima Lemes \\ Endereço: Rua Gabriel Monteiro da Silva, 700, centro. Alfenas, MG. Cep: 37.130-000 - S1 C-123 \\ Email: raquel.lemes@unifal-mg.edu.br \\ APOIO: FAPEMIG
}

Recebido em: 30/05/2014 - Aprovado em: 19/09/2014 - Disponibilizado em: 15/12/2014

RESUMO: As $\beta$-lactamases formam um grande grupo de enzimas capazes de hidrolisar o anel $\beta$-lactâmico de penicilinas, cefalosporinas e monobactâmicos, e Escherichia coli e Klebsiella spp. são as bactérias mais comumente produtoras de ESBL ( $\beta$-lactamase de espectro estendido). A maior frequência de enterobactérias ESBL tem sido em amostras procedentes de pacientes hospitalizados. Objetivamos neste estudo determinar a prevalência da produção de ESBL nas amostras de Klebsiella spp. isoladas de pacientes internados em um hospital universitário, e também estabelecer o índice de isolamento por topografia destes micro-organismos. Foram analisadas 30 cepas de $K$. pneumoniaee 18 deK. ozaenae. O teste para investigação de ESBL foi o método de "screen" que é um método de Dupla Difusão em Disco. A ampliação do halo de inibição em alguma das cefalosporinas ou do aztreonam ou o aparecimento de uma terceira zona irregular (ghostzone) entre o disco de amoxicilina/ác. Clavulânico e o disco de uma das drogas $\beta$ lactâmicas caracterizava a cepa como ESBL. Dentre as 48 amostras analisadas, 40 (83.33\%) foram identificadas como ESBL, sendo $26(86.67 \%)$ de K. pneumoniaee $14(55.56 \%) \mathrm{K}$. ozaenae. Os sítios com maior taxa de isolamento de ESBL foram o SNC e SC (100\% cada), seguidos do TR $(85.71 \%)$, CS (83.33\%), Outros (80\%) e TU (62.5\%). Não houve diferença significativa na análise estatística pelo Qui-quadrado entre as espécies e sítios $(\mathrm{p}>0,05)$. Nenhuma amostra de K. pneumoniaefoi positiva para kpC.

Palavras-chave: ESBL. Klebsiella.Prevalência.Topografia. Hospital Escola.

\section{PREVALENCE OF KLEBSIELLA SPP. ESBL ISOLATED IN A TEACHING HOSPITAL IN SOUTHERN MINAS GERAIS}

\begin{abstract}
The $\beta$-lactamases are a large group of enzymes that hydrolyze $\beta$-lactam ring of penicillin's, cephalosporin's and monobactams, and Escherichia coli and Klebsiella spp. are the most common bacteria producing ESBL ( $\beta$ - lactamase extended spectrum). The higher frequency of ESBL has been enterobacteria samples from hospitalized patients. We aimed in this study to determine the prevalence of ESBL production in samples of Klebsiella spp. isolated from patients in a university hospital, and also establish the insulation index by surveying this micro organisms. Thirty strains of K. pneumoniae and $18 \mathrm{~K}$. ozaenae were analyzed. The test was ESBL to investigating the method of "screen" which is a double diffusion method on Disc. The expansion of the zone of inhibition in any of the cephalosporins or aztreonam or the appearance of a third irregular zone (ghost zone) between the disk of amoxicillin / ác. Clavulanic and a drug disk $\beta$ - lactam characterized the strain as ESBL. Among the 48 samples analyzed, 40 (83.33 $\%)$ were identified as ESBL, $26(86.67 \%)$ of $K$. pneumoniae and $14(55.56 \%)$ K. ozaenae. The sites with higher isolation rate of ESBL were the CNS and SS (100\% each), followed by TR (85.71\%), Blood Stream (83.33\%), other $(80 \%)$ and UT $(62.5 \%)$. There was no significant difference in the statistical analysis by chi-square between species and sites ( $\mathrm{p}>0.05)$. No sample was positive for $K$. pneumoniae $\mathrm{KpC}$.
\end{abstract}

Key words:ESBL. Klebsiella. Prevalence. Topography. School Hospital. 


\section{INTRODUÇÃO}

\section{Resistência bacteriana}

Os mecanismos genéticos que codificam a resistência bacteriana se exteriorizam por seis principais mecanismos bioquímicos de ação: inativação enzimática da droga, alteração da permeabilidade bacteriana à droga, alteração de sistemas de transporte na célula, retirada ativa da droga do meio intracelular, alteração do receptor à droga e modificação do sistema metabólico ativo para a droga e síntese das vias metabólicas alternativas (TAVARES, W. 2002).A inibição ou inativação enzimática produzida pelos micro-organismos é provavelmente o principal mecanismo molecular de resistência microbiana. Foi inicialmente descrita por Abraham e Chaim, em 1940, que demonstraram em extratos de Escherichia coli uma enzima capaz de inativar a ação da penicilina, provocando a sua abertura por hidrólise e transformando o antibiótico em produto inativo (SOUZA JR., M.A.; FERREIRA, E.S.; CONCEIÇÃO, G.C. 2004), conhecida como $\beta$-lactamase.Dentre as $\beta$-lactamases, destacam-se as $\beta$-lactamases de espectro ampliado (Extended-Spectrum $\beta$ lactamase - ESBL), resistentes à penicilina, a todas as cefalosporinas e ao aztreonam, ou seja, são patógenos multirresistentes (SOUZA JR., M.A.; FERREIRA, E.S.; CONCEIÇÃO, G.C. 2004).São sensíveis aos inibidores da $\beta$ lactamases (ácido clavulânico, sulbactam e tazobactam), substâncias semelhantes aos antibióticos, que se unem às $\beta$-lactamases de maneira reversível ou irreversível, impossibilitando sua ação deletéria sobre a droga.O grau de resistência ao $\beta$-lactâmico irá depender da quantidade de enzima produzida, da habilidade dessa enzima em hidrolisar o antimicrobiano em questão e da velocidade com que o $\beta$-lactâmico penetra pela membrana externa da bactéria. A produção de ESBL é mediada por plasmídeos, que conferem ampla resistência aos antimicrobianos que contém o anel $\beta$ lactâmico em sua estrutura.

\section{Bactérias produtoras de ESBL}

Escherichia coli e Klebsiella pneumoniae são as espécies bacterianas mais comumente encontradas produzindo ESBL, porém, a detecção destas enzimas já foi observada em diversas outras espécies de Enterobacteriaceae e Pseudomonadaceae (SOUZA JR., M.A.; FERREIRA, E.S.; CONCEIÇÃO, G.C. 2004). Proteus mirabilis ESBL positivos foram isolados na França (De CHAMPS, C.R. et al., 2000), diversos países da Europa (NIJSSEN, A.S. et al., 2004) e Nova York (SAURINA, G. et al., 2000).As enterobactérias produtoras de ESBLs têm sido isoladas com maior frequência em amostras procedentes de pacientes institucionalizados, internados em unidades de terapia intensiva (KASSIS-CHIKHANI, N.; VIMONT, S.; ASSELAT, K. et al., 2004)e casas de repouso (BRADFORD, P.A.; URBAN， C.; JAISWAL, A. et al.. 1995), porém também podem ser encontradas em amostras de origem comunitária.Klebsiella spp.geralmente 
estão associadas às infecções nosocomiais, principalmente pneumonia e infecções do trato urinário. Em 1998foi relatada pela primeira vezE.coliprodutora de ESBL isolada em urina de paciente idoso de comunidadecom histórico de uso prévio de antibióticos(CORMICAN, M.; MORRIS, D.; CORBETT-FEENEY, G. et al., 1998).

\section{Tipos de enzimas}

Em 1983, foram detectados na Alemanha (Frankfurt) os primeiros isolados clínicos de K.pneumoniae e E.coli resistentes às cefalosporinas de terceira geração. Desde então, têm sido descritas mundialmente numerosas enzimas dos tipos TEM (Temoniera) e SHV (Sulfidril variável) com este fenótipo de resistência (SOUZA JR., M.A.; FERREIRA, E.S.; CONCEIÇÃO, G.C. 2004), que predominam dentre as demais até hoje (SOUZA, G.C., 2007) e estão presentes em $75 \%$ das Enterobacteriaceae isoladas (ZHANEL, G.G. et al., 2008). Atualmente, as ESBLs têm sido classificadas em nove grupos distintos, de acordo com sua sequência de aminoácidos: TEM, SHV, CTX-M (cefotaxima), PER (Pseudomonas extended resistance), VEB (Vietnamese extendedspectrum $\beta$-lactamase), GES (Guiana extended-spectrum $\beta$-lactamase), TLA (TEM$\beta$-lactamase of Amblerclass A), BES (Brazil extended-spectrum $\beta$-lactamase) e OXA (oxacilina). A enzima CTX-M se desenvolveu em E.coli e K. pneumoniaena Espanha, Reino Unido e Rússia.

\section{Fatores de Risco}

Fatores de risco identificados como preditores de organismos de ESBL são: internação anterior, duração prolongada da estadia, aumento da gravidade de doença, internação em UTI, ventilação mecânica, cateter venoso central e cateteres urinários e exposição prévia a antimicrobianos, especialmente a antibióticos $\quad \beta$-lactâmicos(BISSON,G.; FISHMAN, N.O.; PATEL, J.B. et al. 2002) e envolve também a transmissão exógena (CASSETTARI,V.C. et al., 2006).

\section{Epidemiologia}

A maior prevalência de ESBL ocorre na América Latina (44.9\%) mas segundo Jain \& Mondal (2007), na Índia, a alta prevalência de Klebsiella spp. produtora de ESBL é relatado variando de $6 \%$ a $87 \%$.De acordo com publicações na Europa, ESBL cresceu no período entre 1997 e 1999 e entre 2001 e 2002, sendo que os maiores índices se encontram na Grécia (27.4\%) e Portugal (15.5\%) e os menores na Holanda e Alemanha (2 e $2.6 \%$, respectivamente). Na Itália, uma pesquisa realizada em 1999 mostrou que dentre os pacientes hospitalizados, cerca de $6.3 \%$ dos micro-organismos isolados apresentaram ESBL e os agentes produtores foram $K$. pneumoniae e $P$. mirabilis. A maioria das enzimas isoladas foi TEM (92.4\%) e/ou a família SHV detectadas no fenótipo de ESBL (LUZZARO, F.et al., 2006; SÁNCHEZ, M. et al., 2006). O aumento mundial de bactérias resistentes a 
antimicrobianos em infecções adquiridas na comunidade ou em hospitais está ameaçando a capacidade para tratar eficazmente pacientes, ressaltando a necessidade de supervisão contínua, adequada prescrição médica antimicrobiana, controle rigoroso de infecção e novas alternativas de tratamento (LOCKHART, S. R.et al., 2007). Monitorar a prevalência de ESBL é importante no diagnóstico e na definição da terapêutica mais apropriada (LUZZARO, F.et al., 2006).

Diante de tais dados objetivamos neste trabalho investigar a prevalência da produção de ESBL nas amostras de Klebsiella spp., bem como o índice de isolamento por topografia deste micro-organismo em um hospital escola do sul de Minas Gerais.

\section{MATERIAIS E MÉTODOS}

Foram utilizadas 48 amostras viáveis de Klebsiella spp. do banco de culturas do laboratório de microbiologia da Faculdade de Medicina de Itajubá. Destas, 30 amostras foram identificadas por provas bioquímicas e fisiológicas como Klebsiella pneumoniae e 18 como Klebsiella ozaenae. As cepas foram previamente testadas a antimicrobianos conforme o quadro 1.

Quadro 1 -Apresentação do teste de susceptibilidade das amostras de Klebsiella spp. aos antibióticos previamente testados através do Método de disco difusão - Kirby-Bauer.

\begin{tabular}{|l|l|c|c|c|c|c|c|c|}
\hline \multicolumn{1}{|c|}{$\mathbf{N}^{\mathbf{0}}$} & Sítio & ATM & AMC & CFO & CTX & CAZ & IMP & MER \\
\hline K1 & TU & R & S & S & R & R & S & S \\
\hline K2 & SC & - & - & R & - & R & S & S \\
\hline K3 & CS & S & S & R & - & R & S & S \\
\hline K4 & TR & R & R & R & R & R & S & - \\
\hline K6 & CUT & S & S & S & S & S & S & S \\
\hline K7 & TU & S & S & S & S & S & - & - \\
\hline K8 & TR & R & R & R & R & R & S & S \\
\hline K9 & SC & R & S & R & S & R & S & - \\
\hline K10 & SC & R & S & R & S & R & S & S \\
\hline K11 & TU & R & S & R & R & R & - & - \\
\hline K12 & TU & R & S & R & R & R & - & - \\
\hline K13 & OUTROS & R & S & R & R & R & - & - \\
\hline K14 & TU & R & S & R & R & R & - & - \\
\hline K15 & TR & R & S & R & R & R & S & - \\
\hline K17 & CS & R & S & R & R & R & S & - \\
\hline K18 & CS & R & S & R & R & R & S & S \\
\hline K19 & TR & S & R & R & R & R & S & - \\
\hline K20 & TU & R & S & R & R & R & S & S \\
\hline K21 & TU & R & S & S & R & S & S & - \\
\hline K22 & SNC & R & S & R & R & R & S & - \\
\hline K23 & TR & R & R & R & R & R & S & - \\
\hline K24 & TR & R & S & R & R & R & S & - \\
\hline K25 & DESC. & R & S & R & R & R & - & - \\
\hline K26 & TU & R & S & R & R & R & S & S \\
\hline K27 & TU & R & S & R & R & R & S & - \\
\hline K29 & TU & R & S & R & R & R & S & - \\
\hline K30 & TR & R & R & R & R & R & S & - \\
\hline K31 & TR & - & - & R & - & R & S & - \\
\hline K32 & TU & R & S & R & S & R & S & S \\
\hline & & & & & & & \\
\hline
\end{tabular}




\begin{tabular}{|l|l|l|l|l|l|l|l|l|}
\hline K33 & DESC & S & S & S & S & S & - & - \\
\hline K34 & TU & S & S & I & S & S & S & - \\
\hline K36 & TR & S & S & S & S & S & S & - \\
\hline K37 & TR & R & S & R & S & S & - & - \\
\hline K38 & DESC & R & S & R & R & R & - & - \\
\hline K39 & TU & S & S & R & R & R & S & S \\
\hline K42 & CS & R & S & R & R & R & S & S \\
\hline K43 & CS & R & S & R & R & R & S & - \\
\hline K44 & TU & R & S & R & S & S & S & - \\
\hline K45 & TR & S & R & R & R & R & S & - \\
\hline K46 & TU & R & S & R & R & R & S & S \\
\hline K48 & TU & R & R & R & R & R & S & S \\
\hline K49 & TU & R & S & R & S & S & S & - \\
\hline K50 & TR & R & S & R & R & R & S & - \\
\hline K51 & OUVIDO & R & S & R & R & R & S & - \\
\hline K52 & TU & R & S & S & S & S & - & - \\
\hline K53 & TR & R & S & S & S & S & S & - \\
\hline K54 & TR & R & I & R & R & R & S & - \\
\hline K55 & CS & S & S & S & R & R & S & S \\
\hline
\end{tabular}

$\mathrm{S}=$ Sensível $\mathrm{R}=$ Resistente $\mathrm{I}=$ Intermediário

$\mathrm{ATM}=$ AztreonamCFO=CefoxitimaCTX $=$ Cefotaxima

MER=MeropenemAMC=Amoxicilina/Ác.Clavulânico
$\mathrm{CAZ}=$ Ceftazidima

$\mathrm{TU}=$ Trato urinário
$\mathrm{IMP}=\quad$ Imipenem

$\mathrm{CS}=\quad$ Corrente sanguínea

$\mathrm{SC}=$ Sítio cirúrgicoTR $=$ Trato respiratório $\quad \mathrm{CUT} .=$ Cutânea $\mathrm{SNC}=$ Sistema nervoso central

DESC. $=$ Desconhecido

Fonte: Autor, 2014

\section{1 - Teste"screen" para detectar ESBL}

Empregamos o método da aproximação de disco(SOUZA JR, M.A.; FERREIRA, E.S.; CONCEIÇÃO, G.C., 2004) no qual utilizamos uma placa $(150 \mathrm{~mm})$ de ágar Muller-Hinton, inoculamos a cepa a ser testadasuspensa na escala 0,5 de Mac Farland $\left(10^{8} \mathrm{UFC} / \mathrm{ml}\right)$ (DALMARCOet al., 2006). Após, adicionamos no centro daplacao disco de amoxacilina/acido clavulânico e ao redor os antimicrobianos (ceftriaxona, ceftazidime, cefotaxima e aztreonam) (HONORIOet al., 2001) na distância de 20 a 30mm de centro a centro, levando em consideração o disco central. Incubou-se por 18 horas a $35^{\circ} \mathrm{C}$ e após foi realizada a leitura dos halos (DALMARCOet al, 2006). Consideramos positiva a produção de ESBL quando observado um aumento do halo de inibição em alguma das cefalosporinas ou aztreonam ou aparecimento de uma terceira zona("ghostzone"), entre o disco de amoxacilina/acido clavulânico e o disco de uma das drogas $\beta$ lactâmicas (SILVA e SALVINO, 2000).

\section{RESULTADOS}

Dentre as 48 amostras analisadas, 40 $(83.33 \%)$ foram identificadas como ESBL, sendo $26(86.67 \%)$ de K.pneumoniae e 14 (55.56\%) de K. ozaenae(tabela 1). Os sítios com maior taxa de isolamento de ESBL foram o SNC e SC (100\% cada), seguidos do TR (85.71\%), CS $(83.33 \%)$, Outros $(80 \%)$ e TU(62.5\%).Não houve diferença significativa na análise estatística pelo teste de Quiquadrado entre as espécies e sítios ( $\mathrm{p}>0,05)$. Nenhuma amostra de $K$. pneumoniae foi suspeita de serK.pneumoniae produtora de 
carbapenemase $(\mathrm{kpC})$ por apresentarem meropenem.

previamente sensibilidade ao imipenem e

Tabela 1-Distribuição das taxas de ESBL positiva e negativa entre as duas espécies de Klebsiella analisadas segundo o sítio de isolamento.

\begin{tabular}{lcccc}
\hline Sítios & \multicolumn{2}{c}{ K.pneumoniae } & \multicolumn{2}{c}{ K. ozaenae } \\
& \multicolumn{2}{c}{ ESBL } & $+(\%)$ & ESBL \\
& $+(\%)$ & $\varphi(\%)$ & $4(66.67)$ & $2(33.33)$ \\
TU & $11(91.67)$ & $1(8.33)$ & $1(100)$ & 0 \\
CS & $4(80)$ & $1(20)$ & $1(100)$ & 0 \\
SC & $2(100)$ & 0 & $5(71.43)$ & $2(28.57)$ \\
TR & $7(100)$ & 0 & 0 & 0 \\
PELE & 0 & $1(100)$ & $2(100)$ & 0 \\
OUTROS & $2(66.67)$ & $1(33.33)$ & $1(100)$ & 0 \\
SNC & 0 & 0 & $\mathbf{1 4}(\mathbf{7 7 . 7 8 )}$ & $\mathbf{4 ( 2 2 . 2 2 )}$ \\
TOTAL & $\mathbf{2 6 ( 8 6 . 6 7 )}$ & $\mathbf{4 ( 1 3 . 3 3 )}$ & &
\end{tabular}

TU= Trato urinário $\quad \mathrm{CS}=$ Corrente sanguínea $\mathrm{S}=$ Sítio cirúrgico

$\mathrm{TR}=$ Trato respiratório

$\mathrm{SNC}=$ Sistema nervoso central

Fonte: Autor, 2014

\section{DISCUSSÃO}

Dentre as 48 amostras analisadas, 40 (83.33\%) foram identificadas como ESBL, sendo 26 (86.67\%) de K.pneumoniae e 14 (55.56\%), de K. ozaenae.Os dados obtidos nesse estudo revelam uma prevalência, de micro-organismos produtores de ESBL, elevada quando comparados à literatura de estudos realizados em outros países. Sendo o triplo dos índices da Coréia (28.4\%) (ROH et al., 2008), o dobro da América Latina (44.9\%), o triplo da Grécia (27.4\%), 5 vezes superiores aos de Portugal (15.5\%), e muito elevados em relação a Holanda, Alemanha e Itália (2, 2.6 e 6.3\%, respectivamente) (LUZZARO etal., 2006).

A prevalência de cepas produtoras de ESBL em alguns hospitais brasileiros varia em torno de 39\%(BLATT, 2000; MENEZES et al.,
2003). Sendo assim, notamos uma taxa elevada no resultado obtido por esse estudo $(83.33 \%)$, provavelmente pelo fato do hospital analisado ser um hospital escola onde muitos profissionais e acadêmicos entram em contato direto com o paciente e prescrevem diferentes medicamentos, facilitando, portanto o padrão de resistência bacteriana a certos antimicrobianos.Em outros estudos com hospitais universitários ou mesmo em localizações de baixa renda encontramos resultados próximos aos nossos. Em um hospital em Fortaleza encontramos taxas de cepas produtoras de ESBL em torno de 81.24\% (MENEZES et al., 2003), no Rio Grande do Sul taxas de $71.1 \%$ (OLIVEIRA et al., 2009) e em um hospital universitário em São Paulo, 53.8\% (PEREIRA et al., 2003).O combate a estes micro-organismos em 
hospitais pode ser realizado através do conhecimento da microbiota local e a valorização das comissões de controle de infecção hospitalar (SALLES \& SALLES, 2000).A detecção precoce destas bactérias multirresistentes é de suma importância para se instaurar o tratamento adequado e as medidas de isolamento dos pacientes, necessários para se evitar a disseminação destes patógenos(MENEZES et al., 2003).Por topografia, o SNC e SC (100\% cada) foram os de maiores prevalência, seguidos do TR (85.71\%), CS $(83.33 \%)$, outros sítios $(80 \%)$ e TU $(62.5 \%)$. Esses resultados diferem com a literatura onde a K.pneumoniaefoi a causa de 9.7\% das infecções de corrente sanguínea, $11 \%$ das do trato respiratório inferior, $7 \%$ das de pele e mucosas, feridas, e também por $11.7 \%$ das ocorridas nas UTIs. Destas, mais de $50 \%$ eram produtoras de betalactamase (SADER et al., 2001).Todo hospital deve conhecer a sua prevalência (BRADFORD, 2001). Em geral, essas bactérias surgem em unidades de terapia intensiva, seja neonatal, adulta ou pediátrica, devido à alta frequência de uso de medicamentos de amplo espectro nesses locais, concomitante ao estado grave desses doentes que em contato com os demais pode levar a infecção cruzada com frequência razoável nessas unidades, além do fato de poderem adoecer por estes agentes em razão do uso muito frequente de cateteres e sondas de cavidadese tecidos naturalmente estéreis (BLATT, 2000; AMARANTE, 2002).

\section{CONCLUSÃO}

A prevalência e padrão de resistência antimicrobiana de Klebsiella spp.produtora de ESBL neste trabalho, chega próximo a valores de hospitais universitários ou daqueles em locais de baixa renda. Sendo assim é muito importante adetecção de bactérias produtoras de ESBL para só então instaurar o isolamento do paciente e o tratamento eficaz preciso e potente que possibilite a erradicação e evite a disseminação desses patógenos.O uso abusivo de medicamentos de amplo espectro, o contato inadequado com os pacientes sem uso das técnicas de assepsia, assim como a manipulação inadvertida desses pacientes ao se colocar cateteres e sondas de cavidades e tecidos que até então não estavam contaminados, são fontes de disseminação e devem ser evitadas através de treinamento desde o período acadêmico, sendo crucial a conscientização de acadêmicos e profissionais da saúde em nosso meio.

\section{REFERÊNCIAS BIBLIOGRÁFICAS}

AMARANTE, J.M.B. Prevalência de ESBL pode chegar até $100 \%$ das bactérias isoladas em hospitais. Fato Hospitalar, ano III, 7:4-6, 2002.

BISSON, G.; FISHMAN, N.O.; PATEL, J.B. et al. Extended-spectrum b-lactamaseproducing Escherichia coli and Klebsiella species: Infect Control Hosp Epidemiol , 23: 254-60, 2002. 
BLATT, J.M. Mecanismo de resistência e

Detecção das Betalactamases de espectro

Ampliado. NewsLab 40:86-96, 2000.

BRADFORD P.A.; URBAN C.; JAISWAL,

A. et al. SHV-7, a novel cefotaxima

hydrolyzing b-lactamase, identified in

Escherichia coli isolates from hospitalized

nursing home patients. Antimicrob Agents

Chemother, 39: 899-905, 1995.

BRADFORD P.A. Extended-spectrum blactamases in the 21st century:

characterization, epidemiology, and detection of this important resistance threat. Clin

Microbiol Rev; 14: 933-51, 2001.

CORMICAN, M.; MORRIS, D.;

CORBETT_FEENEY, G. et al. Extended

spectrum b-lactamase production and

fluoroquinolone resistance in pathogens

associated with community acquired urinary

tract infection. Diagn Microbiol Infect Dis,

32: 317-9, 1998.

DALMARCO, E.M.; BLATT, S.L.;

CORDOVA, C.M.M. Identificação

Laboratorial de $\beta$-Lactamase de Espectro

Estendido (ESBLs) - Revisão, RBAC, v.

38(3): 171-177, 2006.

DE CHAMPS, C.R.; BONNET, D.; SIROT,

C.C. et al. Clinical relevance of Proteus

mirabilis in hospital patients: a two-year survey. J. Antimicrob. Chemother., 45:537539, 2000.

HONORIO, L.C.; SANTOS, I.B.; ASSIS,

A.M.L. et al.Analise do perfil de resistência de enterobacterias produtoras de betalactamase de espectro ampliado (ESBL) isoladas em João Pessoa, Rev. Bras. Anal. Clin.,33(4):179-182,2001.

JAIN, A.; MONDAL, R. Prevalence \& antimicrobial resistance pattern of extended spectrum $\beta$-lactamase producing Klebsiella spp. isolated from cases of neonatal septicaemia. Indian J Med Res 125:89-94, 2007.

KASSIS-CHIKHANI, N.; VIMONT, S.; ASSELAT, K. et al. CTX-M blactamaseproducing Escherichia coli in longterm care facilities, France. Emerg Infect Dis, 10: 1697-8, 2004.

LOCKHART, S.R.; ABRAMSON, M.A.; BEEKMANN, S.E.et al. Antimicrobial resistance among gram-negative bacilli as causes of infections in intensive care unit patients in the United States between 1993 and 2004. J. Clin. Microbiol. 45:3352-3359, 2007.

\section{LUZZARO, F.; MEZZATESTA,}

M.; MUGNAIOLI,C. et al. Trends in

Production of Extended-Spectrum B-

Lactamases among Enterobacteria of Medical Interest: Report of the Second Italian

Nationwide Survey. J. Clin. Microbiol. 44(5): 
1659-1664, 2006.

MENEZES, E.A. et al. Avaliação do ertapenem frente a bacilos gram negativos produtores de enzima beta lactamase de espectro expandido (ESBL). Rev. Bras. Anal. Clin., 39(3): 189-191, 2007

MENEZES, E. A.; ALVES, E.G.B.; CUNHA, F.A. et al. Avaliação do ertapenem frente a bacilos gram negativos produtores de enzima beta lactamase de espectro expandido (ESBL). Rev. Bras. Anal. Clin., 39(3): 189191, 2007.

MENEZES, E.A. et al.. Frequência de cepas produtoras de enzima beta lactamase de espectro expandido(ESBL) e perfil de susceptibilidade de Klebsiella pneumoniae em hemoculturas no berçário de um hospital de Fortaleza. Rev. Bras. Anal. Clin.40(1):7-11, 2008.

\section{NIJSSEN, A.S.; FLORIJN, M.J.M.;}

BONTEM, F.J. et al.Beta-lactam susceptibilities and prevalence of ESBLproducing isolates among more than 5000 European Enterobacteriaceae isolates. Int. J. Antimicrob. Agents; 24:585-591, 2004.

OLIVEIRA, C.F. et al.. Prevalência das famílias TEM, SHV e CTX-M de $\beta$ lactamases de espectro estendido em Escherichia coli e Klebsiella spp. no Hospital Universitário de Santa Maria, Estado do Rio
Grande do Sul. Rev. Soc. Bras. Med. Trop. 42(5):556-560, 2009.

PEREIRA, A.S. et al.. Avaliação da acurácia de testes laboratoriais para detecção de amostras de Klebsiella pneumoniae produtora de betalactamase de espectro estendido. J. Bras. Patol. Med. Lab. 39:301-308,2003.

ROH, K.H.; UH, Y.; KIM, J.S. et al.First Outbreak of Multidrug-Resistant Klebsiella pneumoniae producing both SHV-12-Type extended-spectrum $\beta$-lactamase and DHA-1Type AmpC $\beta 1$ Lactamase at a Korean Hospital. Yonsei Med J, 49(1):53 - 57, 2008.

SADER, H.S. et al.. Pathogen Frequency and Resistence Patterns in Brazilian hospitals: Sumary of results from three years of de SENTRY Antimicrobial Surveillence Program. Braz. J. Infect. Dis., 5(4):200-214, 2001.

SALLES, J.M.C.; SALLES, M.J.C. Antimicrobianos - Quando indicar Como usar. EditoraUniversitária UFPA, Belém,.p 23-25. 2000 .

SAURINA, G.;QUALE, J. M.;MANIKAL, V. M. et al. Antimicrobial resistance in Enterobacteriaceae in Brooklyn, N.Y.: epidemiology and relation to antibiotic usage patterns. J. Antimicrob. Chemother.; 45:895898, 2000. 
SOUZA, J.R.; M.A.; FERREIRA, E.S.;

CONCEIÇÃO, G.C. Betalactamase de

Espectro Ampliado (ESBL): um Importante

Mecanismo de Resistência Bacteriana e sua

Detecção no Laboratório Clínico, NewsLab, 63:152-174, 2004.
TAVARES, W. Manual de Antibióticos, Quimioterápicos e Anti-infecciosos. Ed. Ateneu, 2002. 